\title{
A Framework for Detecting and Classifying Outbreaks of Gastrointestinal Disease
}

\author{
Kathryn Morrison*, Katia Charland, Anya Okhmatovskaia and David Buckeridge \\ Epidemiology \& Biostatistics, McGill University, Montreal, QC, Canada
}

\section{Objective}

To develop a methodological framework for detecting and classifying outbreaks of gastrointestinal disease on the island of Montreal, with the goal of improving early outbreak detection using simulated surveillance data.

\section{Introduction}

Outbreaks of waterborne gastrointestinal disease occur routinely in North America, resulting in considerable morbidity, mortality, and cost (Hrudey, Payment et al. 2003). Outbreak detection methods generally attempt to identify anomalies in time, but do not identify the type or source of an outbreak. We seek to develop a framework for both detection and classification of outbreaks using information in both space and time. Outbreak detection can be improved by using simulated outbreak data to build, validate, and evaluate models that aim to improve accuracy and timeliness of outbreak detection.

\section{Methods}

To generate outbreak data, we used a previously validated microsimulation model depicting waterborne outbreaks of gastrointestinal disease (Okhmatovskaia et al. 2010). The model is parameterized based on outbreak characteristics such as concentration and duration of contamination, and calibrated to produce realistic outbreak data (e.g., emergency department visits from GI-illness, laboratory reporting to public health) in space and time. We are interested in identifying unique space-time signatures in the data that would allow not only detection, but also classification based on outbreak type. For example, to be able to detect and classify an outbreak as due to a water plant failure versus an food-borne illness based on unique space-time patterns, even though symptoms and temporal outbreak patterns may be similar. For the detection step, we use a hidden Markov model (HMM) that accounts for spatial information through a spatially correlated random effect with an exponential decay. HMMs have been used previously in disease mapping (Green 2002) but not widely in space-time disease outbreak detection. For the classification step, we use a supervised clustering algorithm to classify the outbreak by source (e.g., water plant location) and type (e.g., disease).

\section{Results}

Preliminary results for the detection step show that the HMM can distinguish accurately between regions in an outbreak state versus those in a normal state at each time period. Ongoing work for the de- tection step includes further evaluation of the HMM accuracy as a function of outbreak characteristics. For the classification step, we are evaluating the suitability of different supervised clustering algorithms for identifying the type of outbreak from the HMM results.

\section{Conclusions}

If outbreaks are detected rapidly, interventions, such as boil-water advisories, are available to quickly and effectively limit the human and economic impacts. Traditional public health surveillance systems, however, frequently fail to detect waterborne disease outbreaks. Every disease outbreak has unique characteristics; simulation is the best method to estimate the capacity of syndromic surveillance to more efficiently detect different types of enteric disease outbreaks based on a variety of parameters. Outbreak detection can be improved with advances in data availability, such as syndromic surveillance data that will increase timeliness of detection, and space-time information to allow for simultaneous detection and classification of outbreaks by important characteristics (type of outbreak, source of outbreak).

\section{Keywords}

Syndromic surveillance; Disease outbreak detection; Waterborne disease

\section{Acknowledgments}

This research is supported by the Canadian Institutes of Health Research (CIHR).

\section{References}

Green PJ. (2002). "Hidden Markov Models and Disease Mapping.” Journal of the American Statistical Association 97(460): 1055-1070.

Hrudey S., P. Payment, et al. (2003). "A fatal waterborne disease epidemic in Walkerton, Ontario: comparison with other waterborne outbreaks in the developed world." Water science \& technology 47(3): 7-14

Okhmatovskaia A, Verma AD, Barbeau B, Carriere A, Pasquet R, Buckeridge DL. (2010). A Simulation Model of Waterborne Gastro-Intestinal Disease Outbreaks: Description and Initial Evaluation. AMIA Annual Symposium.

\section{*Kathryn Morrison}

E-mail: kt.morrison@mail.mcgill.ca 